\title{
A NOVEL CHEMICAL AMPLIFICATION TYPE POSITIVE RESIST USING DIPHENYLIODONIUM 9,10-DIMETHOXYANTHRACENE-2-SULPHONATE AS PHOTONITIATOR
}

\author{
KAZUHIKO NAITOH, KIMIO KANAI, TSUGUO YAMAOKA \\ Department of Image Science and Technology, Faculty of Engineering, Chiba \\ University, 1-33 Yayoi-cho, Chiba 260, JAPAN \\ and AKIRA UMEHARA \\ Yoshida-minami Factory, Fuji Photo Film Co.,Ltd., 4000 Kawashiri, Yashida-cho, \\ Haibara-Gun, Shizuoka 421-03, JAPAN.
}

Novolac-diazonaphthoquinone positive photoresists are attractive and widely used in semiconductor fabrication because of their high dry etch resistance and the non-swelling aqueous base development to provide high resolution[1]. Our interests in these areas are concentrated on the improvement of sensitivity which is an important subject on the UV sensitive photoresist systems, which are based on the combined principles of chemical amplification and dissolution inhibition[2]. Upon irradiation, a strong protonic acid is generated from the photoacid generator which can, upon a subsequent bake step, catalyze the conversion of the dissolution inhibition to an aqueous base soluble form. One of the cruicial processing steps is the process of photoacid generation.

It is well known that onium cations, especially diaryliodonium and triarylsulphonium, are useful in the photoinitiation of both radical and cationic polymerizations[3]. On the other hand, in our previous study[4], it was found that p-nitrobenzyl 9,10-dimethoxyanthracene-2-sulphonate photodissociates to generate 9,10-dimethoxyanthracene-2-sulphonic acid, and that this dissociation is considered to proceed via an intramolecular electron transfer from the excited singlet state of 9,10-dimethoxyanthracene moiety to p-nitrobenzyl moiety. By combining diphenyliodonium ion as electron acceptor with 9,10-dimethoxyanthracene-2-sulphonic acid as electron donor, we discovered a novel category of diphenyliodonium salt whose counteranion acts as spectral sensitizer. Herein we report the photochemical behavior of diphenyliodonium 9,10-dimethoxyanthracene-2-sulphonate(DIAS) associated with photoinduced intramolecular electron transfer within ion-pair, in which counteranion acts as both sensitizer and precursor for photoacid generator, and its application to a positive resist which is based on the combined principles of chemical amplification and dissociation inhibition.

DIAS was prepared by the reaction of sodium 9,10-dimethoxyanthracene- 
2-sulphonate(SDAS) with diphenyliodonium chloride(DPI) in a hot water followed by purification by repeated recrystallization from water. The absorption spectrum of DIAS is essentialy the sum of the spectra of SDAS and DPI. No broadening or red-shifting of the spectrum due to ground-state interactions between SDAS and DPI was observed. While the spectral response of DPI extends only to $300 \mathrm{~nm}$, the absorption of DIAS does to longer wavelength than that of DPI. Experimentally it has been confirmed that DIAS reacts by exciting only 9,10-dimethoxyanthracene chromophore with the longer wavelength light.

The fluorescence of SDAS in degassed acetonitrile solution decays with a lifetime of $9.8 \mathrm{~ns}$. The fluorescence lifetime of DIAS is $2.4 \mathrm{~ns}$. With the assumption that the shorter fluorescence lifetime of DIAS, relative to that of SDAS, is due entirely to electron transfer, the rate constant $k_{\circ t^{i n t r a}}$ for intramolecular electron transfer from 9,10-dimethoxyanthracene moiety to iodonium moiety is given by eq1, where $\tau_{2}$ and $\tau_{2}$ are the lifetime of SDAS and DIAS, respectively.

$$
\mathbf{k}_{0 t^{\text {intra }}}=1 / \tau_{z}-1 / \tau_{1}
$$

Using eq. 1 and the lifetimes given above, we find $\mathrm{k}_{e t^{i n t r a}}=3.2 \times 10^{8} \mathrm{~s}^{-1}$ in acetonitrile. DIAS shows irreversible oxidation and reduction waves at $1.09 \mathrm{~V}$ and $-0.23 \mathrm{~V}$ vs SCE, respectively.

Calculation from the Rehm-Weller equation reveals that electron transfer from the excited 9,10-dimethoxyanthracene moiety to iodonium moiety is exothermic by more than $30 \mathrm{kcalmol}^{-1}$.

Intra-ion-pair electron transfer machanism is supported by the results of laser flash photolysis measurements. Excitation of DIAS with the third harmonic pulse( $355 \mathrm{~nm}$, $5 \mathrm{~ns}$ fwhm) of a Q-switched Nd:YAG in oxygen-free acetonitrile solution gives instantaneous formation of transient absorption bands at 310 and $425 \mathrm{~nm}$ essentially identical with that of independently generated 9,10-dimethoxyanthracene-2-sulphonate radical cation. The transient absorption monitored at $425 \mathrm{~nm}$ decayed with first-order kinetics to afford a lifetime of $450 \pm 34 \mathrm{~ns}$ with a similar value at $310 \mathrm{~nm}$ within experimental

a)
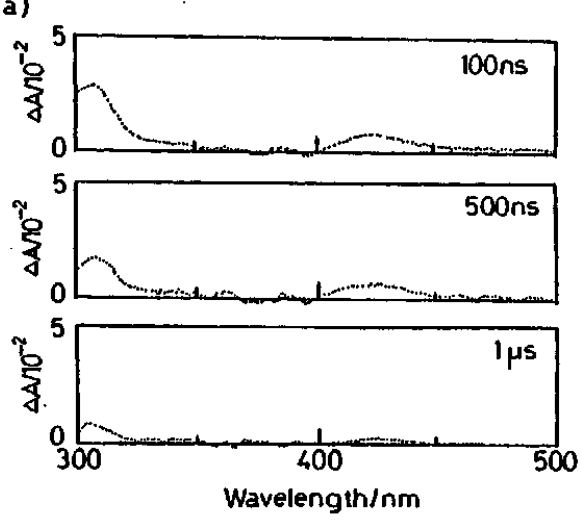

b)

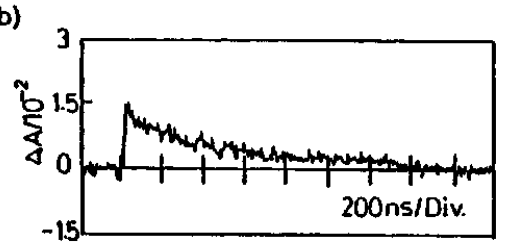

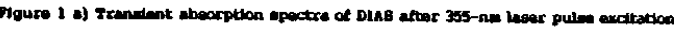
In dopand ecotionitrille solution.

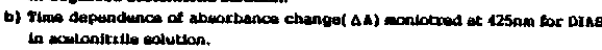
error(Figure 1). 
Irradiation of a degassed acetonitrile solution of DIAS with $365 \mathrm{~nm}$ exciting only by 9,10dimethoxyanthracene moiety leads to formation of 9,10-dimethoxyanthracene-2-sulphonic acid, iodobenzene and benzene which are assigned by electron ionization mass spectroscopy and GC-MS. Acid generation from the photolysis of DIAS was measured by a non-aqueous photomeric method using 3'-diethylamino-7'-methylspiro[isobenzofuran-1(3H), $9^{\prime}-[9 H]$ xanthen]-3-one in acetonitrile standardized with $p$-toluenesulphonic acid. This leuco dye is a satisfactory indicator which is

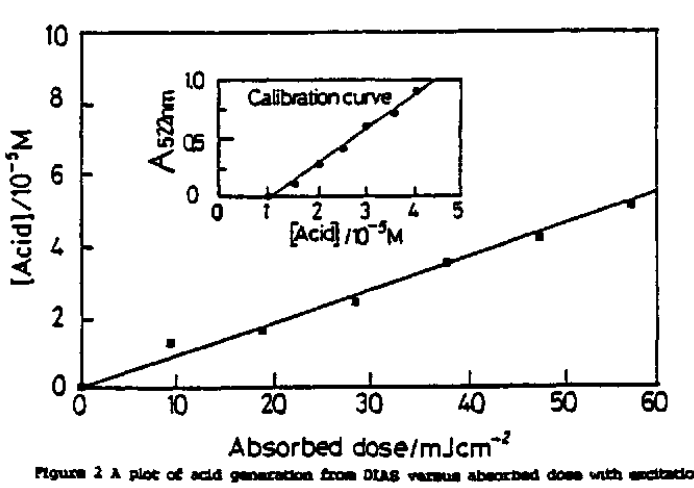

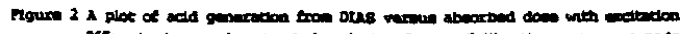

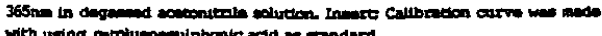
colorless in nature or basic medium and red in acid medium. Quantum yield for photoacid generation is determined to be 0.29 by means of this method(Figure 2).

The mechanism outlined in Scheme I accommodates the experimental findings. The proposed mechanism of the sensitized photolysis of diphenyliodonium salt is that the electron transfer occurs from the excited singlet state of 9,10dimethoxyanthracene moiety to diphenyliodonium ion to form two radical species, 9,10-dimethoxyanthracene- 2-sulphonate cation radical and diphenyliodonium radical. The former radical ultimately forms 9,10-dimethoxyanthracene-2-sulphonic acidby hydrogen abstraction from the solvent, while homolytic cleaveage of the latter gives iodobenzene and benzene as products. This work reveals a new type of diphenyliodonium salt, whose counteranion is sensitizer, that can lead to formation of parent acid and radical species.

Sensitivity of the photopolymer consisting of novolac resin (100 parts) and DIAS(7 parts) as photoacid generator in the absence ( 2 components system, $2 \mathrm{CS}$ ) or presence ( 3 components system, 3CS) of $t$-butyl naphthalene-2-carboxylate(1.3 parts) as typical dissolution inhibitor was measured as follows.

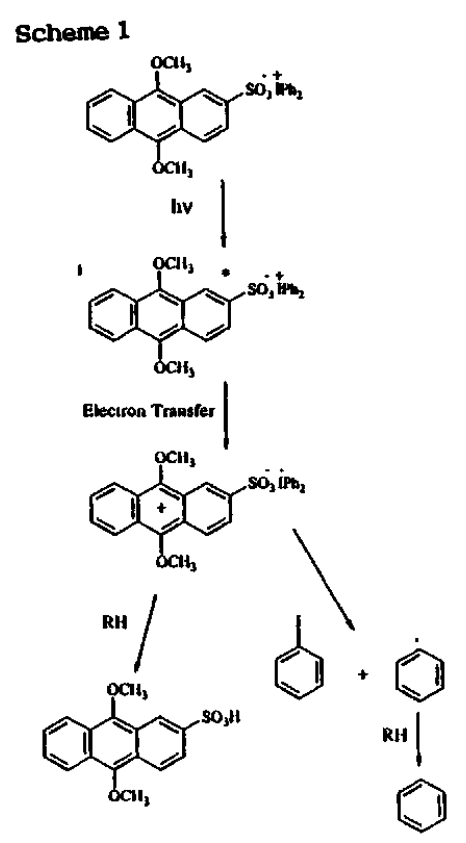

The photopolymer layer coated on an aluminium plate was exposed with $365 \mathrm{~nm}$ light followed by post exposure bake( $P E B$ ) at $80^{\circ} \mathrm{C}$ for several minutes, and then film was developed in a tetramethylammonium hydroxide(TMAH) based aqueous solution for 90 seconds. The minimal incident energy required to solubilize the photopolymer layer completely was defined as the sensitivity. As indicated in Figure 3, the sensitivity for 2CS and 3CS was ploted as a function of 
PEB time. It is interesting that the sensitivity of $3 C$ is evidently dependent on PEB condition, while that of $2 \mathrm{CS}$ is not.

The sensitivity of $2 \mathrm{CS}, 137 \mathrm{~mJ} / \mathrm{cm}^{2}$ is enhanced up to $38 \mathrm{~mJ} / \mathrm{cm}^{2}$ by the aid of the dissolution inhibitor. The sensitivity enhancement of 3CS may be due to the chemical amplification, where the acid photochemically generated from DIAS, catalytically converts the dissociation inhibition to the hydrophilic carboxylic acid during PEB. Thus, PEB has been shown to promote acid-catalyzed the conversion of the dissolution inhibition to an aqueous base soluble form.

In this paper we have described that photochemical behavoir of DIAS which is a novel type of diphenyliodonium salt whose counteranion acts as both sensitizer for iodonium ion and precursor for photoacid generator and that its application to positive resist based on both chemical amplification and dissolution inhibition. Further investigations on silicon wafer are now in progress.

\section{Refierences}

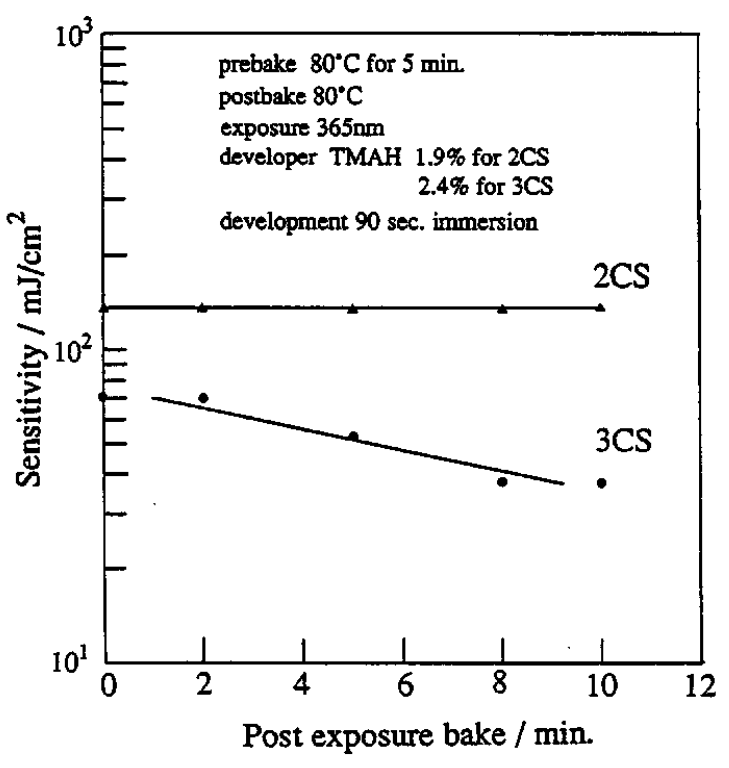

Figure 3 a plot of sensitivity as a tunction of post exposure bake at 80r.

1. M. J. O'Brein, J. V. Crivello; Proc. of SPIE (1988) 920, 42.

2. S. P. Pappas," UV Curing: Science and Technology"; Technology Marketing Corporation, Stanford (1978) Chapter 2.

3. T. Yamaoka, H. Adachi, K. Matsumoto, H. Watanabe and T. Shirosaki, J. Chem. Soc. Perkin Trans. 2 (1990) 1709. 